\title{
Synergistic reduction by metformin and atorvastatin combination of hepato-metabolic changes in rat model of metabolic syndrome
}

\author{
Mervat Samy ${ }^{1}$, Abdel-Motaal Fouda ${ }^{1 *}$, Mohamed-Hesham Daba ${ }^{1}$, Abdel-Rahman Yassin ${ }^{1}$
}

1Department of Clinical Pharmacology,

Mansoura University Faculty of Medicine,

Mansoura 35516, Egypt

${ }^{*}$ Corresponding author: Tel:

+201113974766, Email: fou

daamm@mans.edu.eg

\begin{abstract}
Metabolic syndrome (MetS) with the commonly associated non-alcoholic fatty liver disease (NAFLD) is an increasing health concern and hot topic in medical research. Together with lifestyle modification, metformin (MET) and statins are among drugs with potential therapeutic benefit. The aim of this study is to investigate the potential of synergistic combination of MET and atorvastatin (ATR) in rat model of MetS. Rats were fed on highcarbohydrate, high-fat (HCHF) diet for 16 weeks and either of MET, ATR, or their combination were administered from the beginning of the $9^{\text {th }}$ week to the end of the study. Body weight, insulin resistance, plasma lipids, serum transaminases, TNF- $\alpha$, leptin and adiponectin as well as liver histopathology were assessed. Both MET and ATR worked synergistically to reverse the biochemical and histological abnormalities of MetS and ameliorated steatohepatitis more than their individual effects. The present study clearly demonstrates a synergistic effect of MET and ATR combination to reverse hepato-metabolic abnormalities of MetS/NAFLD in rat model and calls for pursuing subsequent clinical studies to consolidate data at hand.
\end{abstract}

Key words:

metabolic syndrome, metformin, atorvastatin, leptin, adiponectin.

\section{Introduction}

Metabolic syndrome (MetS) is a multiplex of clinical manifestations including hypertension, hyperglycemia, hypertriglyceridemia, diminished highdensity lipoprotein cholesterol (HDL-C), and abdominal obesity [1]. Non-alcoholic fatty liver disease (NAFLD) is widely considered the hepatic manifestation of MetS [2], and results from increased fat accumulation in the liver (steatosis). Pathological picture ranges from non-alcoholic fatty liver (NAFL), with absent hepatocyte injury or fibrosis, to the extreme construct of non-alcoholic steatohepatitis (NASH) in which hepatic steatosis is associated with inflammation, liver cell injury and fibrosis [3]. NASH is a progressive disorder with up to $20 \%$ of patients develops cirrhosis within 10 years [4].

Insulin resistance and associated compensatory hyperinsulinemia are critical etiologic players in the development of MetS [5]. Insulin resistance inhibits glycogenesis and promotes gluconeogenesis and free fatty acid (FFA) release from adipose tissue. The uptake of circulating FFA by hepatocytes is dysregulated, resulting in enhanced synthesis of triglycerides and impaired FFA oxidation [6]. Liver injury involves increased hepatocyte vulnerability to oxidative stress and ensuing lipid peroxidation. By-products of oxidative stress and lipid peroxidation bare forceful chemoattractants of neutrophils with subsequent release of proinflammatory cytokines such as tumor necrosis factor- $\alpha$ (TNF- $\alpha$ ) which in turn exerts a positive feedback loop to increase insulin resistance and neutrophils recruitment. Leptin and adiponectin are two cytokine-like mediators (adipokines) produced by adipose tissue. Leptin facilitates the secretion of TNF- $\alpha$ and other proinflammatory cytokines to increase insulin resistance [7] while, on the flip side, adiponectin typically stimulates fatty acid oxidation and augments insulin sensitivity [8].

Until recently, lifestyle modification has been the only treatment option for MetS. As of today, the FDA has not approved any medications to treat 
MetS; however, metformin (MET) and statins are logical candidates. A meta-analysis study published in 2007 demonstrated that monotherapy with MET leads to normalization of aminotransferases in significant fraction of patients more than dietary regime alone, and also improves steatosis on radiologic imaging [9]. Improvements in liver biochemistry and histology through treatment with statins have been also observed in some patients with NAFLD, although these studies were conducted on relatively small samples of patients [10]. To the best of our knowledge, the combination of MET with atorvastatin (ATR), a competitive inhibitor of HMG-CoA reductase of the statins family, for the treatment of MetS has not been investigated. The purpose of this study however is to study the potential of synergistic combination of MET and ATR in rat model of MetS. We reasoned that the documented improvement of insulin resistance and glycemic control by MET, together with the established role of statins in the management of dyslipidemia may offer a broad spectrum therapeutic benefit in the control of hepato-metabolic abnormalities of MetS.

\section{Material and Methods}

\section{Animal model}

All experiments were conducted according to the Institutional Research Board (IRB) of Mansoura Faculty of Medicine. Seventy male SpragueDawley rats (9-weeks old, 160-190 g body wt) were acclimatized upon arrival for 5 days before experiments in naturally controlled lab of $22 \pm 2{ }^{\circ} \mathrm{C}$ and $12 \mathrm{~h}$ light-dark cycles. Fifty rats were pair-fed to high-carbohydrates, high-fat diet (HCHF) while the remaining 20 rats fed on standard chow diet and served as negative control. The HCHF diet consisted of $395 \mathrm{~g}$ sweetened condensed milk, $200 \mathrm{~g}$ beef tallow, $175 \mathrm{~g}$ fructose, $155 \mathrm{~g}$ powdered rat food, $25 \mathrm{~g}$ Hubble, Mendel and Wakeman salt mixture, and $50 \mathrm{~g}$ water per kilogram of diet. In addition, the drinking water was supplemented with $25 \%$ fructose. After 8 weeks, 10 rats from each side were euthanized to assess the progression of hepato-metabolic changes while the remaining 50 rats were allowed to continue for another 8 weeks to study the effect of drug treatments.

\section{Experimental design}

After the initial 8 weeks of dietetic regime, rats were assigned into 5 groups (each of 10 rats) as follows - (1) negative control group - rats continued on standard chow diet without any drug treatment; (2) positive control group - rats continued on HCHF diet without any drug treatment; (3) MET group - rats continued on HCHF diet and receive MET $200 \mathrm{mg} / \mathrm{kg} / \mathrm{day}$; (4) ATR group - rats continued on HCHF diet and receive ATR $30 \mathrm{mg} /$ $\mathrm{kg} /$ day; and (5) ATR+MET group - rats continued on $\mathrm{HCHF}$ diet and receive ATR plus MET in the same previous doses. All drug treatments were commenced from the beginning of the $9^{\text {th }}$ week to the end of $16^{\text {th }}$ week. Drugs were given daily by oral gavage dissolved in $0.5 \mathrm{ml}$ distilled water at 11:00 AM and all animals were observed daily for food and water intake while the body wt was measured weekly. At the end of 16th week, laparotomy was done under anesthesia following $12 \mathrm{~h}$ period of food deprivation during which rats were given drinking water without fructose supplementation. Livers were harvested and blood samples were collected from the heart and centrifuged at $1500 \times \mathrm{g}$ for $15 \mathrm{~min}$ at $4^{\circ} \mathrm{C}$ to separate serum for biochemical analysis.

\section{Serum lipid profile}

The total cholesterol (TC), triglycerides (TG) and high density lipoprotein cholesterol (HDL-C) were quantified by using colorimetric enzymatic assay kits (Crystal Chem Inc., The Netherland) according to the manufacturers' instructions. The low density lipoprotein cholesterol (LDL-C) was calculated from Friedwald equation: LDL-C $(\mathrm{mg} / \mathrm{dl})=$ TC - (HDL-C + TG/5) [11]. 


\section{Serological assays}

Serum alanine transaminase (ALT) and aspartate transaminase (AST) were measured by using ELISA kits (BioVision, USA). Serum insulin was analyzed by using rat insulin ELISA kits (DRG International Inc., USA). TNF- $\alpha$ leptin, and adiponectin in serum were measured by using ELISA kits (RayBiotech, Inc. USA) according to the manufacturers' protocols.

5. Homeostasis model assessment of insulin resistance (HOMA-IR)

HOMA-IR was used to evaluate insulin resistance by the use of the insulin-glucose product assuming that young adult control rats have an average HOMA-IR analogous to HOMA-IR in humans [12]. The equation was as follows - HOMA-IR = fasting plasma insulin $(\alpha \mathrm{U} / \mathrm{ml})$ * fasting plasma glucose $(\mathrm{mmol} / \mathrm{l}) / 22.5^{[13]}$.

\section{Liver histopathology}

Paraffin-embedded 4 am liver sections were stained with hematoxylin \& eosin (H\&E) and examined by light microscope. We used the activity score developed by the NASH Clinical Research Network to score the extent of steatosis, inflammation, and ballooning [14]. The overall score is calculated as the sum of the scores for steatosis (0-3), lobular inflammation (0-3), and ballooning (0-2), and ranges from 0 to 8 (Table 1). An overall score of $\leq 2$ were diagnosed as normal morphology; a score of $\geq 5$ is considered as a surrogate for the histological diagnosis of NASH while biopsies with scores between 2 and 5 were diagnosed as NAFL.

\section{Statistical analysis}

Statistical analyses were performed using the SPSS v22 (SPSS Inc., Chicago, USA). Data were presented as mean \pm SD. Comparisons between means were performed as appropriate by using the Student's t-test or one-way ANOVA followed by Tukey post hoc test. P-values of 0.05 or less were considered significant.

\section{Results}

1. Hepato-metabolic characteristics after 8 weeks of $\mathrm{HCHF}$ diet

Table 2 summarizes biological variables after 8 weeks of HCHF diet while Fig 1 illustrates changes in body wt during 16 weeks of the study. After 8 weeks, rats fed on HCHF diet had marked increase in their body wt, serum glucose, insulin, and HOMA-IR as compared with negative control rats $(P<0.001$ for all). There was also deterioration of serum lipid profile as observed with elevated TC, TG, and LDL-C ( $P<0.001$ for all), and lowering of HDL-C $(P<0.05)$ as compared to control rats. Serum transaminases, leptin, and TNF-? were also higher in HCHF fed rats, while adiponectin level was significantly lower than control rats $(P<0.001$ for all). Histological examination of the livers of HCHF fed rats revealed mild macrovesicular steatosis, few inflammatory cell infiltration and ballooning with an overall score of $4.4 \pm 0.8$ (Fig 6-B).

\section{Effect of drug treatments after 16 weeks}

At the end of the 16th week, rats treated with MET alone or in combination with ATR had significantly lower body weights as compared with nontreated $\mathrm{HCHF}$ fed rats $(\mathrm{P}<0.01)$ while those treated with ATR alone had non-significant reduction of their body weights (Fig 1). There was also significant attenuation of serum insulin, glucose and HOMA-IR values by either of MET or ATR, however, improvements of these glycemic indices were less marked in rats treated with ATR than in rats treated with MET. On the flip side, the maximal improvement in glycemic indices was observed with combined MET and ATR treatments (Fig 2).

After 16 weeks, there was significant deterioration of serum TC, TG, LDL-C and HDL-C in rats fed on HCHF diet $(P<0.001$ for all, Fig 3). Treatment with MET significantly lowered both serum TC and LDL-C $(P<0.05)$ while treatment with ATR signifi- 
cantly lowered serum levels of all the 4 parameters with higher significance levels than those obtained with MET treatment on TC and LDL-C. Rats treated with combined MET and ATR had the maximal improvements in serum TC, TG, LDL-C and HDL-C compared to either MET or ATR alone (Fig 3).

Liver transaminases, AST and ALT, reflecting the degree of liver injury were significantly higher in $\mathrm{HCHF}$ fed rats than negative control rats $(P<0.001$, Fig 4). Rats treated with MET or ATR solely had significantly lower levels of hepatic enzymes as compared with non-treated rats. Cotreatment with MET and ATR significantly lowered the enzyme levels more than these obtained with either of drugs alone (Fig 4).

Fig 5 represents the effect of MET, ATR, and their combination on serum levels of TNF- $\alpha$, leptin and adiponectin. After 16 weeks there was marked upsurge of serum TNF- $\alpha$ and leptin with reciprocal decrease of serum adiponectin in non-treated $\mathrm{HCHF}$ fed rats. MET treatment partially decreased
TNF- $\alpha$ and leptin but not adiponectin, while ATR treatment partially restored TNF- $\alpha$ and adiponectin but not leptin. Rats received combined MET and ATR treatments had significant improvement of serum levels of the three cytokines.

\section{Histopathological changes}

In comparison with normal liver tissue seen in rats fed on standard chow diet, livers of rats fed on HCHF diet for 16 weeks showed progressive macrovesicular steatosis, inflammatory cell infiltration and ballooning with an overall score of $6.3 \pm 1.16$ (Fig 6-C). Treatment of rats with either MET or ATR improved the histological deterioration as seen in reduced steatosis, inflammation, and ballooning with slightly better results seen with ATR over MET treatment (overall scores $3.7 \pm 0.51$ and $4.2 \pm 0.54$ respectively, $P<0.05$, Fig $6-D$ and $E$ ). The combined MET and ATR treatment has significantly attenuated the histological changes more than either of MET or ATR alone with an overall score of $3.2 \pm 0.5(P<0.05$ and $P<0.001$ vs. ATR and MET respectively, Fig 6-F).

Table 1. Components of nonalcoholic fatty liver disease activity score [14]

\begin{tabular}{lll}
\hline Item & Definition & Score \\
\hline Steatosis & $<5 \%$ & 0 \\
& $5 \%-33 \%$ & 1 \\
& $>33 \%-66 \%$ & 2 \\
& $>66 \%$ & 3 \\
Lobular inflammation & No foci & 0 \\
& $<2$ foci per $200 \times$ field & 1 \\
& $2-4$ foci per $200 \times$ field & 2 \\
Ballooning & $>4$ foci per $200 \times$ field & 3 \\
& None & 0 \\
& Few balloon cells & 1 \\
& Many cells/prominent ballooning & 2 \\
\hline
\end{tabular}


Table 2. Biological and serological variables of rats fed on HCHF diet after 8 weeks.

\begin{tabular}{lll}
\hline & Negative control & HCHF diet \\
\hline Body wt $(\mathrm{g})$ & $198.5 \pm 39.61$ & $255.1 \pm 54.90 \dagger$ \\
Serum glucose $(\mathrm{mmol} / \mathrm{l})$ & $4.77 \pm 0.88$ & $6.82 \pm 1.32 \ddagger$ \\
Serum insulin $(\mu \mathrm{U} / \mathrm{ml})$ & $7.1 \pm 1.65$ & $11.03 \pm 2.49 \ddagger$ \\
HOMA-IR & $1.48 \pm 0.19$ & $3.34 \pm 0.72 \ddagger$ \\
TC $(\mathrm{mg} / \mathrm{dl})$ & $105.3 \pm 37.43$ & $198.2 \pm 46.28 \ddagger$ \\
TG $(\mathrm{mg} / \mathrm{dl})$ & $95.33 \pm 20.48$ & $120.17 \pm 26.71^{*}$ \\
HDL-C $(\mathrm{mg} / \mathrm{dl})$ & $57.50 \pm 17.64$ & $39.31 \pm 12.44^{*}$ \\
LDL-C (mg/dl) & $38.67 \pm 14.54$ & $134.0 \pm 36.95 \ddagger$ \\
ALT $(\mathrm{U} / \mathrm{l})$ & $5.5 \pm 1.26$ & $19.4 \pm 4.61 \ddagger$ \\
AST $(\mathrm{U} / \mathrm{l})$ & $9.8 \pm 2.32$ & $22.80 \pm 5.63 \ddagger$ \\
Leptin $(\mathrm{pg} / \mathrm{ml})$ & $77.50 \pm 21.34$ & $347.33 \pm 47.67 \ddagger$ \\
Adiponectin $(\mathrm{ng} / \mathrm{ml})$ & $64.0 \pm 16.76$ & $17.67 \pm 4.35 \ddagger$ \\
TNF- $(\mathrm{pg} / \mathrm{ml})$ & $2.72 \pm 0.87$ & $6.53 \pm 1.96 \ddagger$ \\
\hline
\end{tabular}

Data are represented as mean $\pm \mathrm{SD}$ of 10 rats.

Significance levels $* \mathrm{P}<0.05, \dagger \mathrm{P}<0.01, \$ \mathrm{P}<0.001$ vs. negative control ( $t$-test).

Fig 1. Body wt changes of control, non-treated $\mathrm{HCHF}$ fed rats and treated rats during 16 weeks duration of the study.
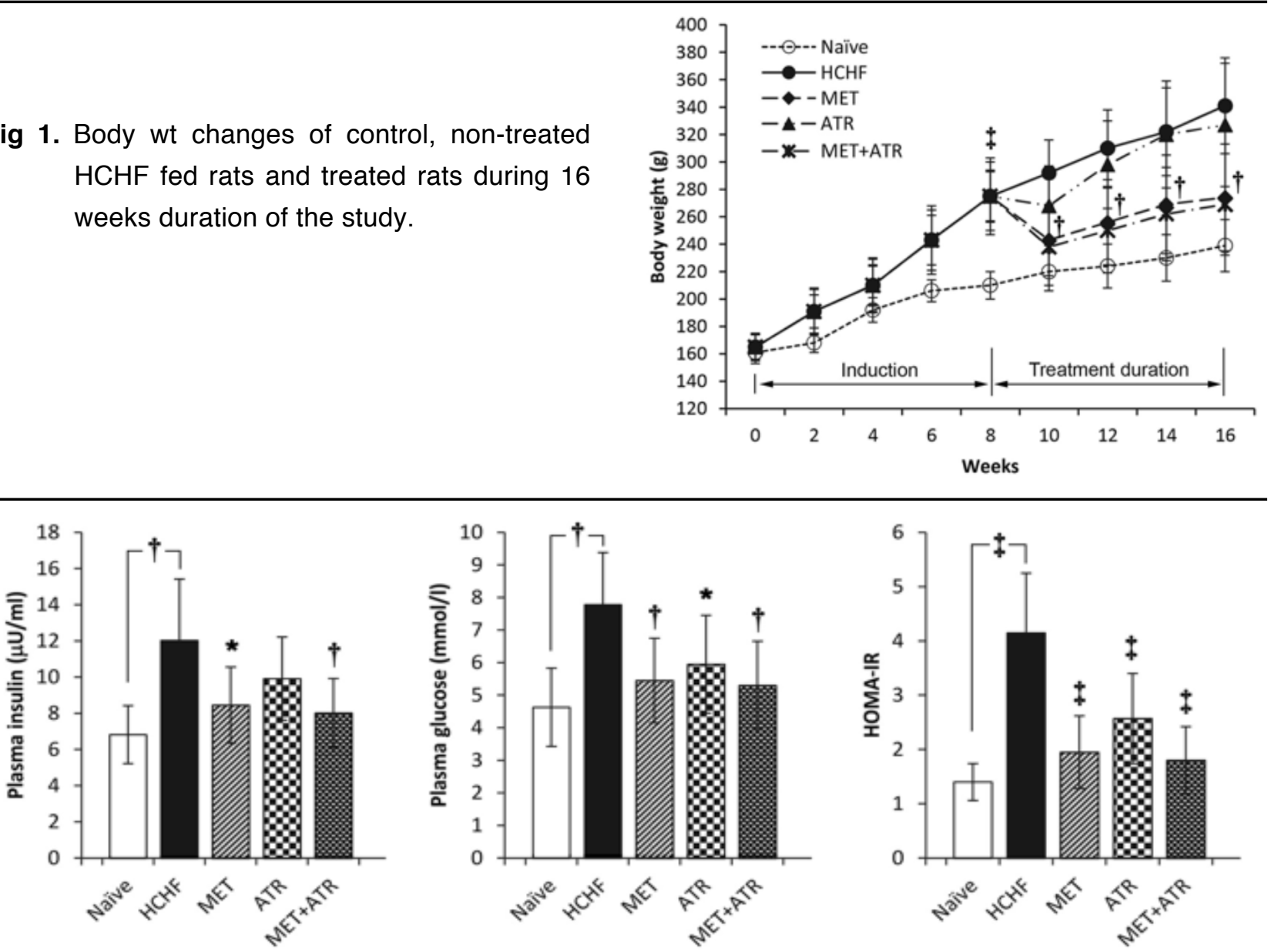

Fig 2. Effect of MET, ATR and their combination on the glycemic control in HCHF fed rats after 16 weeks (Significance levels: ${ }^{*} \mathrm{P}<0.05$; $\uparrow \mathrm{P}<0.01 ; \ddagger \mathrm{P}<0.001$ vs HCHF positive control rats - one way ANOVA of 10 rats). 
Fig 3. Effect of MET, ATR and their combination on serum lipid profile in $\mathrm{HCHF}$ fed rats after 16 weeks (Significance levels: ${ }^{*} \mathrm{P}<0.05 ; \dagger \mathrm{P}<0.01 ; \ddagger \mathrm{P}<0.001$ vs $\mathrm{HCHF}$ positive control rats - one way ANOVA of 10 rats).
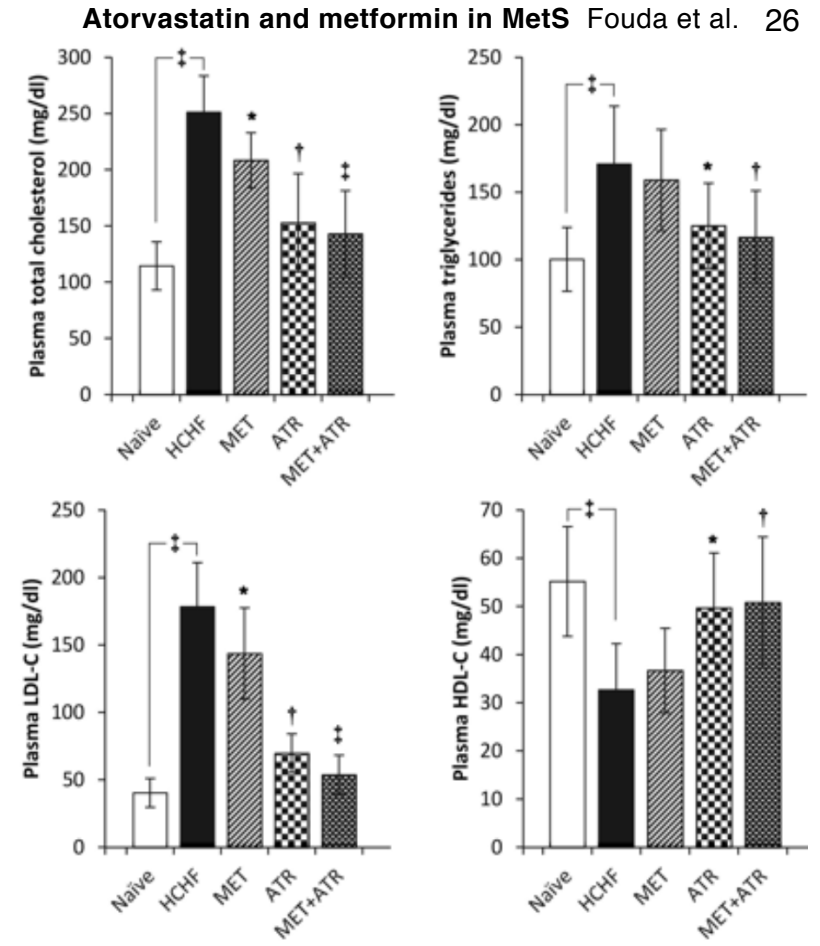

Fig 4. Effect of MET, ATR and their combination on serum AST and ALT in HCHF fed rats after 16 weeks (Significance levels: ${ }^{*} \mathrm{P}<0.05$; $\dagger \mathrm{P}<0.01$ vs HCHF positive control rats - one way ANOVA of 10 rats).
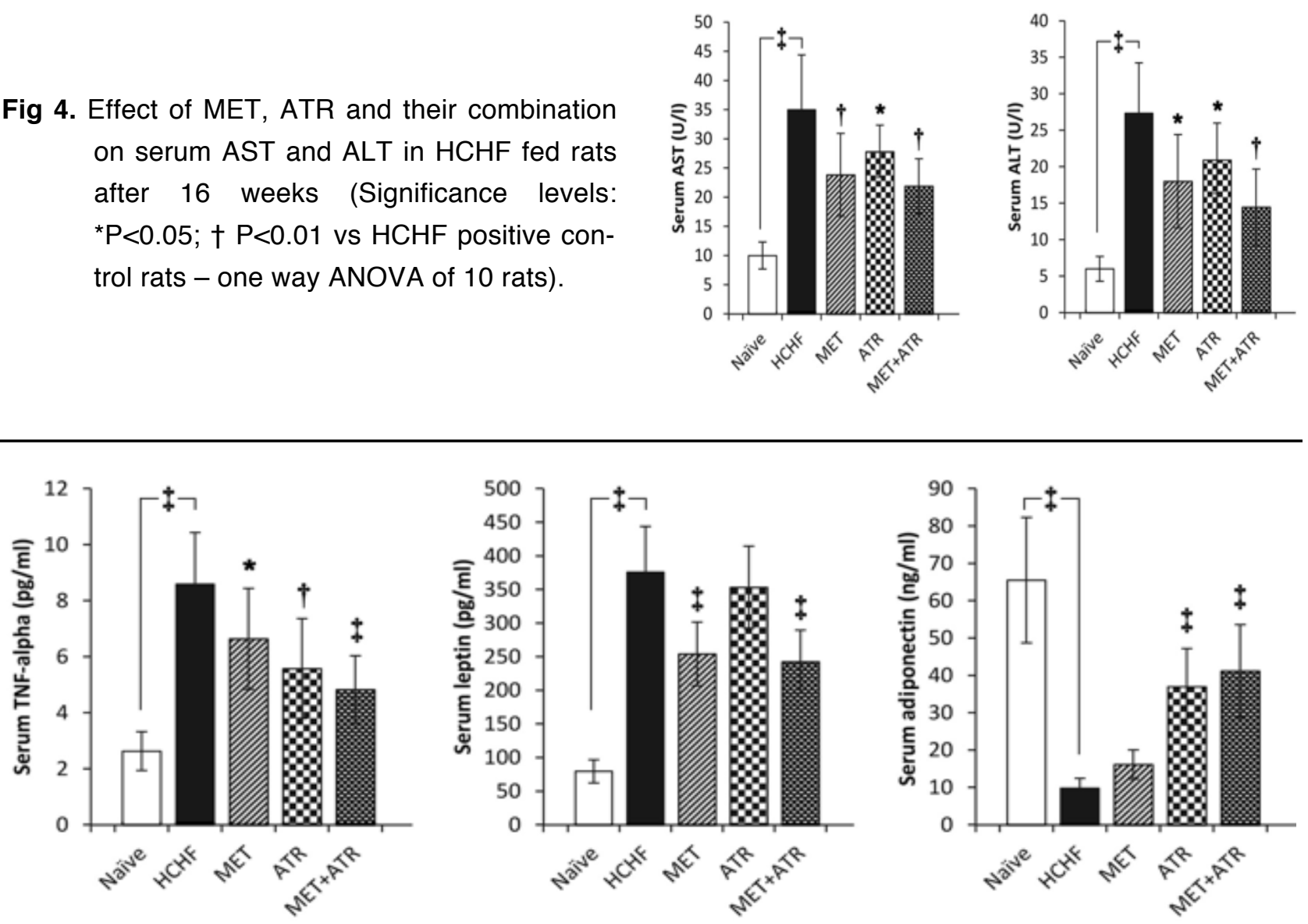

Fig 5. Effect of MET, ATR and their combination on TNF-?, leptin, and adiponectin in HCHF fed rats after 16 weeks (Significance levels: ${ }^{*} \mathrm{P}<0.05 ; \dagger \mathrm{P}<0.01 ; \ddagger \mathrm{P}<0.001$ vs $\mathrm{HCHF}$ positive control rats - one way ANOVA of 10 rats). 

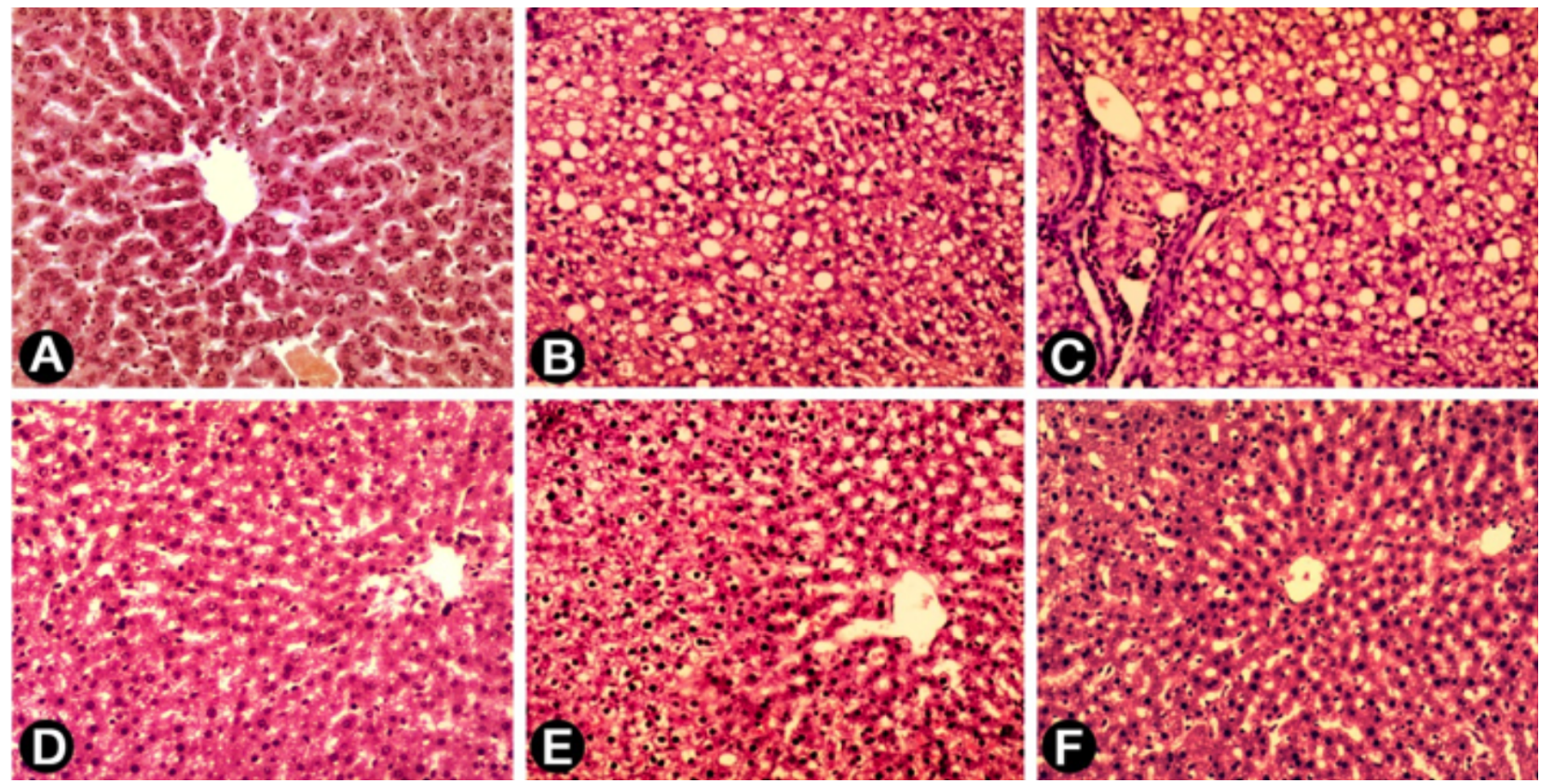

Fig 6. H\&E stained liver sections from control and treated rats. Normal morphology as seen in control rats after 16 weeks (A). Mild macrovesicular steatosis with few inflammatory cell infiltration and ballooning seen in $\mathrm{HCHF}$ fed rats after 8 weeks (B). Progressive steatosis with marked inflammatory cell infiltration seen in HCHF fed rats after 16 weeks (C). Reduced histological changes in MET and ATR treated rats ( $D$ and $E$ respectively). Maximal improvement was seen in rats treated with MET and ATR combination (F). (X100).

\section{Discussion}

MetS and commonly associated NAFLD is an area of ongoing medical research and a number of drugs are being investigated to find out safe and effective treatment. Although therapeutic benefit of MET and statins in MetS are documented in both experimental and clinical studies but information on the value of their combination in MetS/ NAFLD is still lacking. Our results presented herein clearly demonstrate good synergistic effect by MET and ATR in improving both hepato-metabolic and morphological picture of the liver in rat replica of MetS. The benefit of using animal models of MetS in research is the ability to examine biochemical, functional, and histological changes which is difficult to conduct in humans. From the above finding, HCHF diet model used in this study correlates with other studies which reported that rat model of MetS shows most of pathogenic findings known to be responsible for the occurrence of MetS/NAFLD associated with obesity and insu- lin resistance in humans [15].

The body of evidence accumulated during the past 20 years has shown that the overall antihyperglycemic effect of MET is largely due to the inhibition of hepatic gluconeogenesis; however, a detailed explanation of MET mechanism of action in obesity, insulin resistance and MetS is still not fully understood. It seems plausible that part of MET pleotropic effects is due to increase in the AMP/ATP and ADP/ATP ratios [16] leading to stimulation of AMP kinase (AMPK), a primary cellular energy sensing enzyme [17]. However, this observation has been argued in experiments with hepatocytes deficient in either AMPK or its upstream activating cytosolic proteins [18]. The longterm favorable pleiotropic effects of MET may result not only from the AMPK-dependent mechanism but also due to stimulation of other signaling pathways such as increased nitric oxide (NO) synthesis consequent to activation of endothelial NO synthase (eNOS) [19], and suppression of oxida- 
tive stress through inhibition of mitochondrial electron transport chain [20].

As with our findings, there is a substantial body of evidence that obesity-insulin resistance progression is positively correlated with an increase in pro-inflammatory cytokines such as TNF- $\alpha$ and leptin, and reduced plasma concentration of 'protective' adipokines, especially adiponectin [21-22]. MET might be able to positively interfere with leptin and TNF- $\alpha$ production and partially recover adiponectin level and improve 'adiponectin resistance' present in obese individuals and patients with MetS. In human studies, MET treatment of obese adolescents with insulin resistance improved inflammatory activity by eliciting a reduction in TNF- $\alpha$ concentration and preventing the decline in serum adiponectin concentration [23].

Although several studies have reported beneficial effects of statins in patients with MetS [24, 25], but clinicians are often concerned about prescribing statins for patients with elevated liver enzymes [26]. Surprisingly, we found that ATR has reduced aminotransferase levels in our rat model of MetS/ NAFLD. Similar findings were also reported in patients with NAFLD in a number of studies [27-28] which provide our results more consolidation. As far as we know, very few studies have examined statins and MET combination in MetS [29]; moreover, most of these studies were focused mainly on the cardiovascular events, and even so, data obtained by these studies are inconsistent. More specifically, information on the effect of MET and ATR combination in patients with MetS and/or experimental models is lacking.

As expected in this study, improvement of the lipid profile (TC, TG, LDL-C, and HDL-C) was marked in the ATR treated rats as opposed by the marked improvement of insulin resistance (assessed by HOMA-IR) in the MET treated rats. In addition, after 8 weeks of intervention with either MET or ATR, a desirable decline of inflammatory markers, particularly TNF- $\alpha$, was noted. We also observed that while the effects of the two drugs were differential on leptin and adiponectin concentrations, but the combined treatments triggered greater outcome in the reduction of TNF- $\alpha$ and leptin on one hand, and recovery of serum adiponectin on the other hand. These serological findings have been further confirmed by morphological assessment of liver sections where we found that $\mathrm{HCHF}$ fed rats treated with MET and ATR solely has improved the overall histological score as evidenced by decreased steatosis, inflammation, and ballooning with somewhat slightly favorable result with ATR than MET. This finding seems reasonable since the major ATR mechanism of action is directed toward reduction of cholesterol biosynthesis and modulation of lipid metabolism [30], consequently reduction of hepatic steatosis. Similar results were also obtained in some previous studies [27-28]. A more important finding presented herein is that co-treatment with MET and ATR could efficiently ameliorate histological deterioration seen in HCHF fed rats more significantly than monotherapy with either MET or ATR alone. This finding was supported by biochemical and serological data of the present study.

A major limitation of this study is that we did not investigate effects related to the cardiovascular complications of MetS such as blood pressure, myocardial functions or vascular abnormalities as we aimed mainly at studying the hepato-metabolic function. Another limitation is that we did not investigate the effect of drug combination at the molecular levels. Despite these limitations, we think that this study might provide useful information on the beneficial outcome of MET and ATR in experimental MetS which could be easily reproduced in the clinical setting on patients with MetS since MET is commonly used for long-term by patients with obesity, and type-2 diabetes with significant margin of safety; likewise, ATR is given for longterm control of dyslipidemia with good tolerability by most patients. Larger clinical studies would be indeed more useful to consider limitations of data 
at hand and to consolidate the value of this drug combination in the management of patients with MetS.

\section{Conflict of interest statement}

The authors declare they have no conflict of interest.

\section{Acknowledgement}

The authors are deeply grateful to Dr. Amr El Karef, associate professor of pathology, Mansoura Faculty of Medicine, for his generous help in histopathology assessment of liver sections in this study.

\section{References}

1 Kaur J. A Comprehensive Review on Metabolic Syndrome. Cardiol Res Pract. 2014;2014:1-21.

2 Boppidi H. Nonalcoholic Fatty Liver Disease: Hepatic Manifestation of Obesity and the Metabolic Syndrome. Postgrad Med. 2008;120(2).

3 Rinella ME. Nonalcoholic fatty liver disease: a systematic review. JAMA(Systematic review). 2015; 313 (22): 2263-73.

4 McCulough, Arthur J. The clinical features, diagnosis and natural history of nonalcoholic fatty liver disease. Clin Liver Dis. 2004; 8(3): 521-33.

5 Reaven G. Why a cluster is truly a cluster: insulin resistance and cardiovascular disease. Clin Chem. 2008; 54(5):785-7.

6 Saltiel AR, Kahn CR. Insulin signalling and the regulation of glucose and lipid metabolism. Nature. 2001; 414(6865):799-806.

7 likuni N, Lam QL, Lu L, Matarese G, La Cava A. Leptin and inflammation. Curr Immunol Rev. 2008; 4(2): 70-79.

8 Handelsman Y. Metabolic syndrome pathophysiology and clinical presentation. Toxicol Pathol. 2009;37(1):18-20.

9 Angelico F, Burattin M, Alessandri C, Del Ben $M$, Lirussi $F$. Drugs improving insulin resistance for non-alcoholic fatty liver disease and/ or non-alcoholic steatohepatitis. Cochrane Database Syst Rev. 2007; (1):CD005166.

10 Chalasani N, Younossi Z, Lavine J, Diehl A, Brunt E, Cusi K, Charlton M, Sanyal A. The Diagnosis and Management of Non-alcoholic Fatty Liver Disease: Practice Guideline by the American Gastroenterological Association, American Association for the Study of Liver Diseases, and American College of Gastroenterology. Gastroenterology. 2012; 142(7):15921609.

11 Friedewald WT, Levy RI, Fredrickson DS. Estimation of the concentration of low-density lipoprotein cholesterol in plasma, without use of the preparative ultracentrifuge. Clin Chem. 1972;18:499-502.

12 Matthews DR, Hosker JP, Rudenski AS, NayIor BA, Treacher DF, Turner RC. Homeostasis model assessment: insulin resistance and beta cell function from fasting plasma glucose and insulin concentrations in man. Diabetologia. 1985; 28: 412- 419.

13 Antunes LC, Elkfury JL, Jornada MN, Foletto $\mathrm{KC}$, Bertoluci MC. Validation of HOMA-IR in a model of insulin-resistance induced by a highfat diet in Wistar rats. Arch Endocrinol Metab. 2016; 60(2):138-42.

14 Kleiner DE, Brunt EM, Van Natta M, Behling C, Contos MJ, Cummings OW, Ferrell LD, Liu YC, Torbenson MS, UnalpArida A, Yeh $M$, McCullough AJ, Sanyal AJ. Design and validation of a histological scoring system for nonalcoholic fatty liver disease. Hepatology. 2005; 41: 1313-1321.

15 Senaphan K, Kukongviriyapan U, Sangartit W, Pakdeechote $\mathrm{P}$, Pannangpetch $\mathrm{P}$, Prachaney $P$. Ferulic acid alleviates changes in a rat model of metabolic syndrome induced by highcarbohydrate, high-fat diet. Nutrients. 2015;7:6446-64.

16 Hawley SA, Ross FA, Chevtzoff C, Green KA, Evans A, Fogarty S, Towler MC, Brown LJ, Ogunbayo OA, Evans AM, Hardie DG. Use of cells expressing gamma subunit variants to 
identify diverse mechanisms of AMPK activation. Cell Metab. 2010;11:554-65

17 Hardie DG, Ross FA, Hawley SA. AMPK: a nutrient and energy sensor that maintains energy homeostasis. Nat Rev Mol Cell Biol. 2012;13:251-62

18 Foretz M, Hébrard S, Leclerc J, Zarrinpashneh E, Soty M, Mithieux G, Sakamoto K, Andreelli $F$, Viollet $B$. Metformin inhibits hepatic gluconeogenesis in mice independently of the LKB1/ AMPK pathway via a decrease in hepatic energy state. J Clin Invest. 2010;120:2355-69

19 Davis BJ, Xie Z, Viollet B, Zuo M. Activation of the AMP-activated kinase by antidiabetes drug metformin stimulates nitric oxide synthesis in vivo by promoting the association of heat shock protein 90 and endothelial nitric oxide synthase. Diabetes. 2006;55:496-505

20 Ouslimani N, Peynet J, Bonnefont-Rousselot $D$, Thérond $P$, Legrand $A$, Beaudeux J. Metformin decreases intracellular production of reactive oxygen species in aortic endothelial cells. Metabolism. 2005;54:829-34

$21 \mathrm{Kwon} \mathrm{H}$, Pessin JE. Adipokines mediate inflammation and insulin resistance. Front Endocrinol. 2013;4:71

22 Yadav A, Kataria MA, Saini V, Yadav A. Role of leptin and adiponectin in insulin resistance. Clin Chim Acta. 2013; 417:80-4.

23 Evia-Viscarra ML, Rodea-Montero ER, Apolinar-Jiménez E, Muñoz-Noriega N, GarcíaMorales LM, Leaños-Pérez C, FigueroaBarrón M, Sánchez-Fierros D, Reyes-García JG. The effects of metformin on inflammatory mediators in obese adolescents with insulin resistance: controlled randomized clinical trial. J Pediatr Endocrinol Metab 2012;25:41-9

24 Ott C, Schmieder RE. The role of statins in the treatment of the metabolic syndrome. Curr $\mathrm{Hy}-$ pertens Rep. 2009;11(2):143-9.

25 Jialal I, Devaraj S. Statin therapy for the metabolic syndrome: the evidence base. Metab Syndr Relat Disord. 2009;7(5):393-6.

26 Rzouq FS, Volk ML, Hatoum HH, Talluri SK, Mummadi RR, Sood GK. Hepatotoxicity fears contribute to underutilization of statin medications by primary care physicians. Am J Med Sci. 2010; 340(2):89-93.

27 Hyogo H, Tazuma S, Arihiro K, Iwamoto K, Nabeshima $\mathrm{Y}$, Inoue $\mathrm{M}$, Ishitobi $\mathrm{T}$, Nonaka $\mathrm{M}$, Chayama K. Efficacy of atorvastatin for the treatment of nonalcoholic steatohepatitis with dyslipidemia. Metabolism. 2008; 57 (12):1711-8.

28 Georgescu EF, Georgescu M. Therapeutic options in non-alcoholic steatohepatitis (NASH). Are all agents alike? Results of a preliminary study. J Gastrointestin Liver Dis. 2007; 16 (1):39-46.

29 Bulcão C, Ribeiro-Filho FF, Sañudo A, Roberta Ferreira SG. Effects of simvastatin and metformin on inflammation and insulin resistance in individuals with mild metabolic syndrome. Am J Cardiovasc Drugs. 2007;7(3):219-24.

30 Stancu C, Sima A. Statins: mechanism of action and effects. J Cell Mol Med. 2001;5 (4):378-87. 